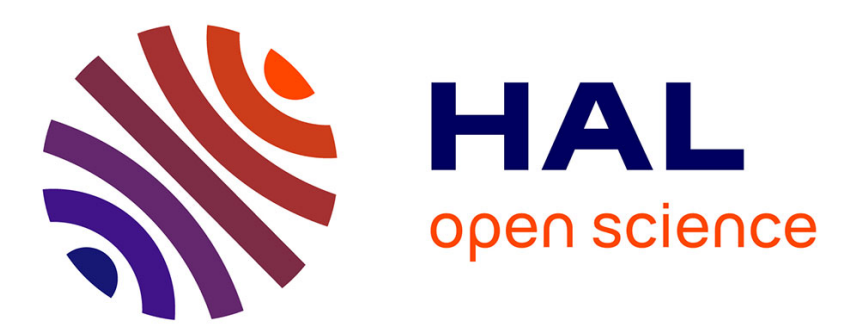

\title{
Wave optics version of photoreflectance signal formation in thermal wave experiments with solids
}

\author{
K. Muratikov
}

\section{To cite this version:}

K. Muratikov. Wave optics version of photoreflectance signal formation in thermal wave experiments with solids. Journal de Physique IV Proceedings, 1994, 04 (C7), pp.C7-777-C7-780. 10.1051/jp4:19947183 . jpa-00253245

\section{HAL Id: jpa-00253245 https://hal.science/jpa-00253245}

Submitted on 1 Jan 1994

HAL is a multi-disciplinary open access archive for the deposit and dissemination of scientific research documents, whether they are published or not. The documents may come from teaching and research institutions in France or abroad, or from public or private research centers.
L'archive ouverte pluridisciplinaire HAL, est destinée au dépôt et à la diffusion de documents scientifiques de niveau recherche, publiés ou non, émanant des établissements d'enseignement et de recherche français ou étrangers, des laboratoires publics ou privés. 


\title{
Wave optics version of photoreflectance signal formation in thermal wave experiments with solids
}

\author{
K.L. Muratikov \\ A.F. Ioffe Physicotechnical Institute of the Academy of Sciences of Russia, Politehnicheskaya 26, \\ 19402I St. Petersburg, Russia
}

\begin{abstract}
A common approach to the determination of the photoreflectance signal within the framework of wave optics is developed. It is shown that different modes of operation of the thermal wave photoreflectance microscope are possible.
\end{abstract}

\section{INTRODUCTION}

In recent years there has been increasing interest in the development and application of a photoreflectance thermal wave method. It has been successfully used as a powerful technique for thermal wave imaging and diagnostics of solids, imaging of integrated circuits and semiconductors [1]. The main advantages of this method are the nondestructive and noncontact type of imaging and the ability to provide high spatial resolution.

A further progress in this direction to a great extent is connected with the correct interpretation of the data obtained by the photoreflectance method. The influence on the photoreflectance signal of the sample surface perturbation produced by the optically generated thermal waves is usually investigated within the framework of geometric optics approach [1]. The influence of the sample surface perturbation on the probe beam in this approach is described in terms of deviation in the direction of the reflected probe beam. The effects of influence of thermal and plasma waves on the reflectance coefficient can also be taken into account in this case by means of intensity modulation of the reflected probe beam.

The purpose of this paper is to develop a common wave optics approach to the calculation of the photoreflectance signal taking into account both the thermal bumping and the modulation of the reflection coefficient. The wave optics approach for the conditions in which the radius of the probe beam is much larger than the size of the region perturbed by the pump laser beam has been developed by Favro, Kuo and Munidasa [2,3]. It was shown that under certain conditions an interferometric effect can play an important role in the process of photoreflectance signal formation.

\section{GREEN'S METHOD OF CALCULATION OF REFLECTED PROBE BEAM}

For the determination of the photoreflectance signal on the base of wave optics it is necessary to know an electric field distribution in the reflected probe beam. It can be found with the help of Green's boundary value theorem. Let us consider in this paper a simplified situation in which the reflection coefficient is independent from the polarization oft the probe beam light. In this case Green's expression 
can be reduced to a scalar form

$$
E^{(r)}\left(\boldsymbol{r}^{\prime}\right)=\frac{1}{4 \pi} \int d s\left[E^{(r)}(\boldsymbol{r}) \frac{\partial}{\partial n}\left(\frac{e^{i k\left|r-r^{\prime}\right|}}{\left|\boldsymbol{r}-\boldsymbol{r}^{\prime}\right|}\right)-\frac{\partial E^{(r)}(\boldsymbol{r})}{\partial n} \frac{e^{i k\left|r-r^{\prime}\right|}}{\left|\boldsymbol{r}-\boldsymbol{r}^{\prime}\right|}\right]
$$

where $E\left(\mathbf{r}^{\prime}\right)$ and $E(\mathbf{r})$ are the amplitudes of electric fields of the probe laser beam in the position of registration and on the surtace of the sample respectively, $k=2 \pi / \lambda, \lambda$ is the wavelength of the probe laser, $n$ is the vector normal to the sample surface $(|n|=1)$.

The electric field of the reflected probe beam can be determined from expression (1) if the electric field and its normal derivation are known on the surface of the sample. For the case with a small radius of curvature of the perturbed surface the Rayleigh boundary conditions [2] can be used

$$
E^{(r)}(r)=V(\boldsymbol{r}) E^{(i)}(\boldsymbol{r}), \quad \frac{\partial E^{(r)}(r)}{\partial n}=-V(r) \frac{\partial E^{(i)}(r)}{\partial n},
$$

where $V(\mathbf{r})$ is the reflection coefficient, $E^{(i)}(r)$ is the electric field of the incident probe beam.

These approximated boundary conditions are valid for the most experimental conditions with thermal waves. Using expression (1) and boundary conditions (2) one obtains

$$
E^{(r)}(r)=\frac{1}{4 \pi} \int d s V(r) \frac{\partial}{\partial n}\left[\frac{E^{(i)}(r) e^{i k\left|r-r^{\prime}\right|}}{\left|r-r^{\prime}\right|}\right] .
$$

Let us consider the process of signal formation in thermal wave photoreflectance microscope on the base of expression (3). For simplicity only the case with the normal incident probe beam will be investigated in this paper. Geometric arrangement of photoreflectance thermal wave microscope of this type is shown in Fig. 1. If the sample surface is determined by the equation $z=h(x, y)$ then using the ordinary expression for the normal derivative and differential element of the surface equation (3) can be expressed in the form

$$
E^{(r)}\left(\boldsymbol{r}^{\prime}\right)=\Delta E_{\perp}^{(r)}\left(\boldsymbol{r}^{\prime}\right)+E_{z}\left(\boldsymbol{r}^{\prime}\right)
$$

where

$$
\Delta E_{+}^{(r)}\left(r^{\prime}\right)=-\frac{1}{4 \pi} \int d x \int d y V(\boldsymbol{r})\left\{\frac{\partial h}{\partial x} \cdot \frac{\partial}{\partial x}\left[E^{(i)}(r) \frac{e^{i k\left|r-r^{\prime}\right|}}{\left|r-r^{\prime}\right|}\right]+\frac{\partial h}{\partial y} \cdot \frac{\partial}{\partial y}\left[E^{(i)}(\boldsymbol{r}) \frac{e^{i k\left|r-r^{\prime}\right|}}{\left|r-r^{\prime}\right|}\right]\right\},
$$

$$
E_{z}^{(r)}\left(\boldsymbol{r}^{\prime}\right)=\frac{1}{4 \pi} \int d x \int d y V(r) \frac{\partial}{\partial z}\left[E^{(i)}(\boldsymbol{r}) \frac{e^{i k\left|r-r^{\prime}\right|}}{\left|\boldsymbol{r}-\boldsymbol{r}^{\prime}\right|}\right]
$$

The field $\Delta E_{\perp}{ }^{(r)}$ depends only on the derivatives in $x$ and $y$ directions. The field $E_{z}^{(r)}$ depends on the derivative in $z$ direction. Let us consider the behavior of these fields more 'detailed. Because of the deformations of the sample surface and reflection coefficient modulations in photothermal experiments are usually small enough we will perform the analysis in the first order of the perturbation theory. The expression for the field $\Delta E_{1}^{(r)}$ is already of the first order due to the existence of the derivatives from $h$. Under these

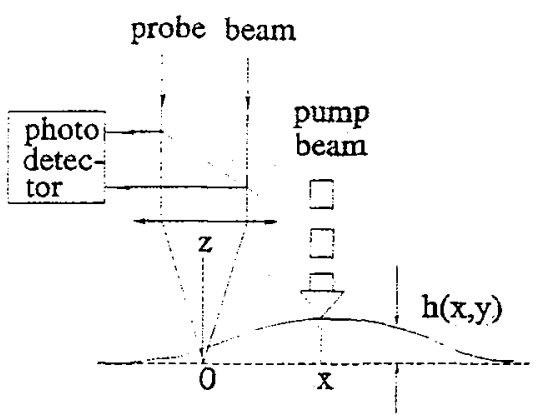

Fig. 1. The optical scheme of the photoreflectance microscope. conditions all other values in this expression can be taken on the unperturbed surface which coincides with $(x, y)$ plane. In the expression for the field $E_{z}{ }^{(c)}$ all values should be taken on the deformed surface 
and then presented in the form corresponding to the first order of the perturbation method. In this case it can be expressed as

$$
E_{z}^{(r)}\left(r^{\prime}\right)=E_{0}^{(r)}\left(r^{\prime}\right)+\Delta E_{z}^{(r)}\left(r^{\prime}\right)
$$

where $E_{0}{ }^{(r)}$ is the field in the probe bean reflected from the unperturbed sample surface, $\Delta \mathrm{E}_{\mathrm{z}}{ }^{(\mathrm{r})}$ is the perturbed part of the probe beam field.

Because of the photoreflectance signal is connected with the second term in equation (5) we shall consider only the behavior of this component of $\mathrm{E}_{\mathrm{z}}{ }^{(\mathrm{r})}$ field.

\section{CALCULATION OF THE PHOTOREFLECTANCE SIGNAL}

For the determination of the photoreflectance signal it is necessary to know the a.c. component of the reflected probe beam intensity in the photodetector plane. It is determined by the total perturbation of the electric field in the reflected beam

$$
\Delta E^{(r)}\left(r^{\prime}\right)=\Delta E_{-}^{(r)}\left(r^{\prime}\right)+\Delta E_{z}^{(r)}\left(r^{\prime}\right)
$$

The perturbation of the probe beam intensity then can be written in the form

$$
\Delta I\left(x^{\prime \prime}, y^{\prime \prime}\right)=E_{0}^{(r)}\left(x^{\prime \prime}, y^{\prime \prime}\right) \cdot \Delta E^{*}\left(x^{\prime \prime}, y^{\prime \prime}\right)+E_{0}^{(r) *}\left(x^{\prime \prime}, y^{\prime \prime}\right) \cdot \Delta E\left(x^{\prime \prime}, y^{\prime \prime}\right),
$$

where $\Delta E(x, y)$ is the perturbation of the electric field of the reflected probe beam in the photodetector plane. If the distribution of intensity in the photodetector plane is known then the photoreflectance signal can be obtained. In the case of bi-cell photodetector it may be written as

$$
S=K \int_{0}^{\infty} d x^{\prime \prime} \int_{-\infty}^{\infty} d y^{\prime \prime} \Delta l\left(x^{\prime \prime}, y^{\prime \prime}\right)
$$

where $\mathrm{K}$ is the proportionality coefficient of the light intensity at the photodetector and the electric signal.

For the determination of the photoreflectance signal with the help of equations $(6),(7)$ it is necessary to know the field in the unperturbed reflected probe beam. The complex representation of the Gaussian beam can be used for the unperturbed reflected probe beam [3].

The perturbed reflected beam is determined by equation (4). In the paraxial approximation it can be represented in the form of Fresnel-Kirchhoff integral. In the case of modulation of the reflectivity coefficient $V$ it leads to the following result

$$
\Delta E_{\zeta}\left(x^{\prime}, y^{\prime}\right)=\frac{1}{i \lambda} \frac{e^{i k z^{\prime}}}{z^{\prime}} \int d x \int d y \Delta E_{V, e j f}(x, y) \exp \left(i k \frac{\left(x-x^{\prime}\right)^{2}+\left(y-y^{\prime}\right)^{2}}{2 z^{\prime}}\right),
$$

where

$$
\Delta E_{V, e f f}(x, y)=\frac{i \lambda}{4 \pi} \Delta V(x, y) E^{(i)}(x, y)\left[\frac{1}{z_{1}-i z_{0}}-2 i k+\frac{i k\left(x^{2}+y^{2}\right)}{2\left(z_{1}-z_{0}\right)}\right], \quad z_{0}=k w_{0}^{2},
$$

$w_{0}$ is the waist size (at $z=z_{1}$ ) of the incident probe beam. The similar equation can be obtained for the 
perturbation of the field produced by the surface deformation. For the fields $\Delta \mathrm{E}_{\perp}{ }^{(r)}$ and $\Delta \mathrm{E}_{z}{ }^{(r)}$ one obtains

$$
\begin{gathered}
\Delta E_{1, e f f}(x, y)=\frac{i \lambda V_{0} E^{(i)}}{4 \pi}\left(\frac{\partial^{2} h}{\partial x^{2}}+\frac{\partial^{2} h}{\partial y^{2}}\right) \\
\Delta E_{z, e f f}(x, y)=\frac{i \lambda V_{0}}{2 \pi} h E^{(i)}\left\{\frac{1}{\left(z_{1}-i z_{0}\right)^{2}}-\frac{2 i k}{z_{1}-i z_{0}}-2 k^{2}+\frac{\left(x^{2}+y^{2}\right)}{\left(z_{1}-i z_{0}\right)^{2}}\left[k^{2}+\frac{i k}{z_{1}-i z_{0}}-\frac{k^{2}\left(x^{2}+y^{2}\right)}{8\left(z_{1}-i z_{0}\right)^{2}}\right]\right\} .
\end{gathered}
$$

With the help of equations (8),(9), and (10) one can determine the perturbation of the field in the reflected probe beam. It should be noted that for a slightly focused probe beam these equations coincide with the similar ones presented in papers $[3,4]$.

Let us consider in this paper the situation in which the photodetector is situated in the image plane and the probe beam is focused on the sample surface. In this case it is possible to replace the integral in equation (7) by the same integral in $(x, y)$ plane and to put $z_{1}=0$. It is seen from equations (6), (9), and (10) that the photoreflectance signal produced by the fields $\Delta E_{\perp, \text { eff }}^{(r)}$ and $\Delta E_{z, e f f}(r)$ in this case is equal to zero. Only the modulation of reflectivity gives the contribution to the photoreflectance signal and it has the following form

$$
S_{V}=K \int_{0}^{\infty} d x \int_{-\infty}^{\infty} d y I(x, y) \Delta R(x, y)\left[1-\frac{1}{2 k z_{0}}+\frac{x^{2}+y^{2}}{4 z_{0}^{2}}\right],
$$

where $I$ is the intensity of the incident probe beam, $\Delta R$ is the modulation of reflectivity for the intensity of the probe beam.

The last two terms in expression (11) are rather small compared with the first one therefore it has the form usual for the photoreflectance signal connected with the modulation of reflectivity.

More complicated situation takes place when the probe laser beam is not strictly focused on the sample surface or the photodetector is not situated in the image plane of the lens. In this case all types of perturbations contribute to the photoreflectance signal. However, the detailed investigation of these cases is not within the framework of this paper and will be published somewhere.

\section{CONCLUSION}

The obtained results show that different modes of the photoreflectance microscope are possible. Only the modulation of reflectivity contributes to the signal in the case of the probe beam strictly focused on the sample surface and detector situated in the image plane. In other cases there is additional influence of the sample surface deformation on the photoreflectance signal. In all cases the photoreflectance signal can be determined in the wave optics approach on the base of Green's boundary value theorem.

\section{References}

[1] Opsal J., Rosencwaig A., and Willenborg D.L., Appl. Opt. 22 (1983) 3169-3176.

[2] Favro L.D. and Munidasa M., Rev. Prog. Quant. NDE 8A (1989) 635-640.

[3] Kuo p.K. and Munidasa M., Appl. Opt. 29 (1990) 5326-5331.

[4] Rytov S.M., Vvedenie v statisticheskuyu phisiku (Nauka, Moscow, 1966) 404p.(in Russian). 